\title{
Characteristics of Merging Occupants in a Staircase
}

\author{
NAOHIRO TAKEICHI ${ }^{1}$, YOSHIYUKI YOSHIDA ${ }^{1}$, TOMONORI SANO ${ }^{2}$, \\ TAKESHI KIMURA ${ }^{3}$, HITOSHI WATANABE ${ }^{4}$, and YOSHIFUMI OHMIYA ${ }^{5}$ \\ ${ }^{1}$ Takenaka Corporation \\ 1-1-1, Shinsuna \\ Koto-ku, Tokyo, 136-0075, Japan \\ ${ }^{2}$ Faculty of Human Sciences \\ Waseda University \\ ${ }^{3}$ Advanced Research Institute for Science and Engineering \\ Waseda University \\ ${ }^{4}$ Faculty of Science and Engineering \\ Waseda University \\ ${ }^{5}$ Department of Architecture, Faculty of Science and Technology \\ Tokyo University of Science
}

\begin{abstract}
The purpose of this study was to investigate the effects of merges on crowds in a staircase and the ease of merging with variations in crowd density, directions of merge, and whether the door joining a hallway to a staircase was opened or closed. The results led to the following conclusions: 1) merging is easier at lower population density; 2) merging is easier if the direction of the merging crowd is same as the direction of the crowd in the staircase; and 3) merging is easier if the door is initially opened, and the evacuation speed is decreased by $30 \%$ when the door is initially closed.
\end{abstract}

KEYWORDS: evacuation, travel, density, merging, flow

\section{INTRODUCTION}

In case of fire in a high-rise or multiple-occupancy building, many evacuees utilize a staircase at once. In the staircase, merging occurs between the evacuees descending from upper floors and ones entering the staircase at lower floor levels. This merging may have an impact on escape time not only on the fire floor but also on the time for evacuating the entire building. However, research about the effects of merging in staircases is scarcely observed [1].

In the notification methods codified into Japanese Building Law, the merge ratio between the number of persons on the stairs and those entering depends on the ratio of door widths leading to the escape routes for each crowd [2]. Many network model simulations for evacuation also employ the ratio of door widths to calculate the merging ratio. However, these ratios are not verified by experimental data, nor are the open- or closed-state of the door or direction of crowd flow considered. To identify the impact of merging, we performed some experiments in a real staircase in which many people merged under a variety of circumstances.

\section{METHODOLOGY}

The tests were performed using a real staircase in a building. We divided the test subjects into two groups of crowds: the first group descended from the upper floor, and the second group merged with the first group from a hallway through a door. We used video cameras 
mounted overhead and stopwatches which were distributed to each person prior to the tests to record the time taken to merge the second group with the first. Then, we performed an analysis based on the population density on the staircase, the direction of merging and the state of the door (opened or closed).

\section{Facility}

The geometry of a staircase is shown in Fig. 1. The width of the stair was $1400 \mathrm{~mm}$, which is the minimum legal width of inside stairs for schools and large stores commonly built in Japan. The staircase had two doors on the both sides of the landing area. Three video cameras were positioned around the landing, aiming down from above the test subjects as they reached the landing.

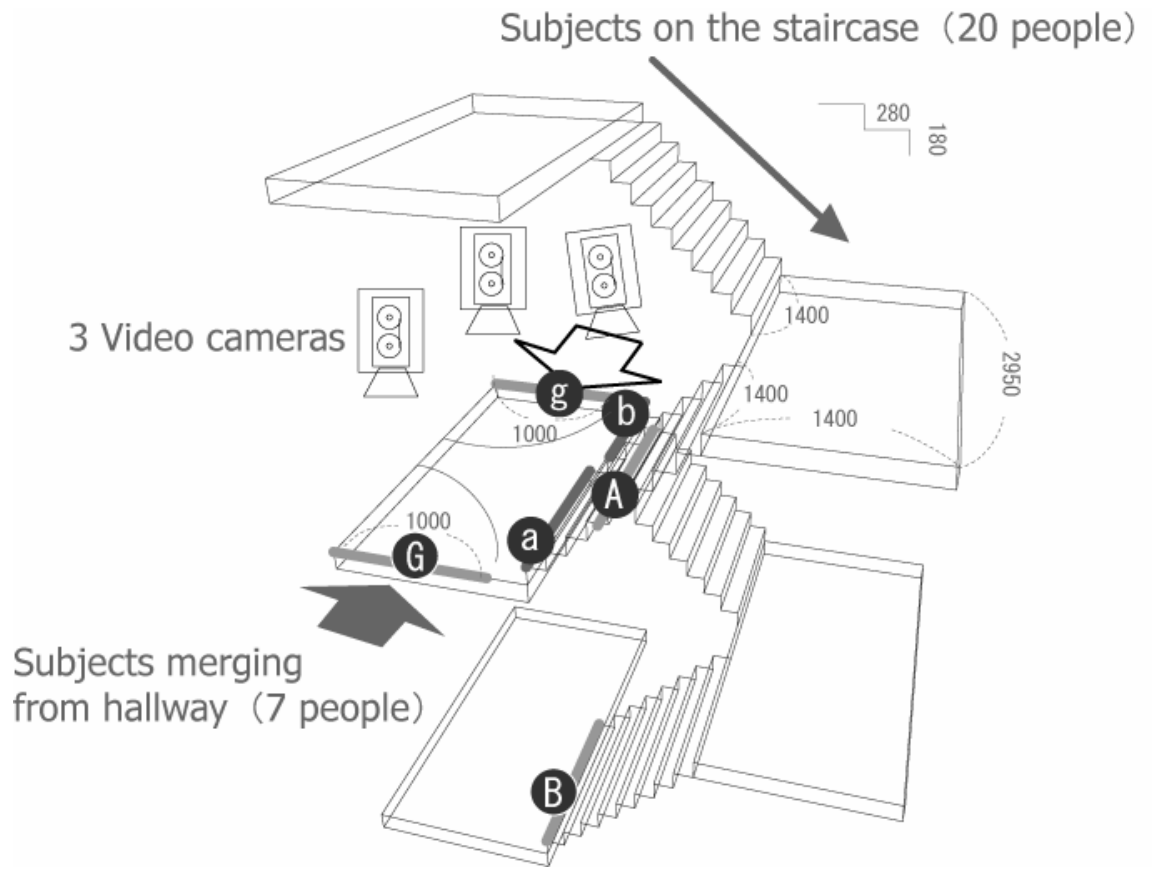

Fig. 1. Test setup.

\section{Test Subjects}

The test subjects were divided into two groups: one group consisted of 14 men and 6 women who were standing in the staircase; the second group consisted of 7 men who were standing in front of the staircase door. All subjects were college students.

\section{Procedure}

1. The test subjects were divided into two groups: the staircase group, which descended from 3 steps above the landing area in the staircase (20 people); and the merging group, which proceeded from a hallway (7 people). Each person was provided with a stopwatch to measure the merging time while video cameras recorded the scene. To identify the group to which each subject belonged, subjects wore differently-colored caps representing each of the groups. 
2. The starter cued the staircase group to start descending. After their speed became steady, the starter cued the merging group to start entering the staircase. Test subjects were instructed to walk at their ordinary speed. There were no bottlenecks in the staircase so that test subjects could maintain their speeds when no merging was occurring.

3. Five people in the staircase group measured both the time of entering the landing area at point "a", and the time elapsed between passing points "a" and "b," where the landing area ends. The remaining 15 people timed only the latter (Fig. 1).

4. Five people in the merging group measured both the time of passing the entrance of staircase named point "G/g"; and time elapsed between passing points "G/g" and "b". The distinction between "G" and "g" is made to denote the relative direction of flow from entrance to the staircase. In the case of entering from point " $G$," the direction of the merging group was the same as the direction of descending group. On the other hand, in the case of entering from point "g," the merging subjects walked against the staircase group.

5. To vary population density, the subjects in the staircase were arranged as follows:

Pattern 1 (P1): 1 line down the center of the steps

Pattern 2 (P2): 2 lines

Pattern 3 (P3): 3 lines which completely filled the space to artificially raise the density

Pattern 1' (P1'): 1 line along the outside of the steps to leave space on the inner side.

Those initial line formations are indicated in Fig. 2. The subjects were not ordered to maintain their initial line formation. Occasionally, the lines became unstable at the merge point.
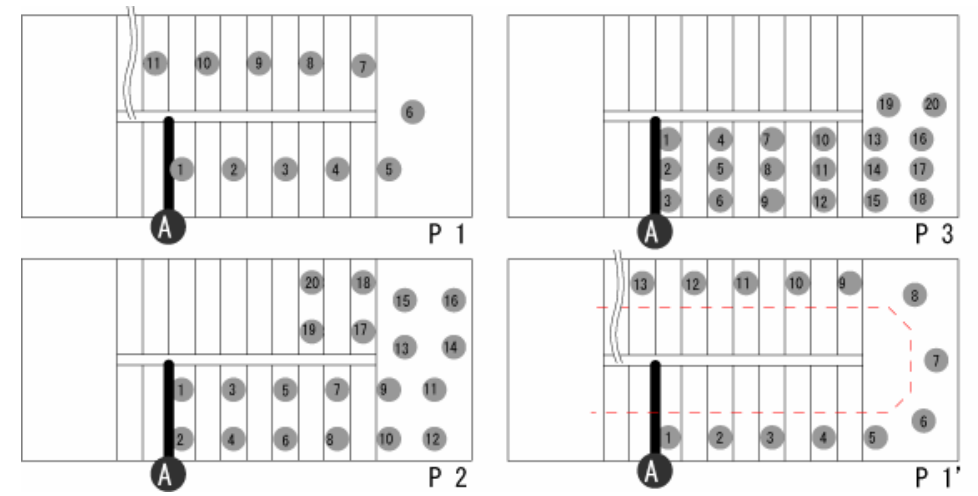

Fig. 2. Overhead view of initial line formations.

6. After the staircase group descended, the merging group entered the landing area of the staircase. Three types of merging were tested: both directions of merging and no merging. Two states of the entrance door obstructing the merging group were also tested, namely "initially opened" and "initially closed." Each test was performed once or twice. 
7. The subjects of the test groups answered a questionnaire regarding the speed and ease of merging after each test.

\section{RESULTS}

\section{Comparison of Flows to Population Density}

The flows were measured at the entering door point "G/g." The rates were calculated as follows:

Flow rate $=\frac{N}{w \times\left(t_{1}-t_{2}\right)}$

where $N$ is the number of people entering the staircase, $w$ is the width of stairs, $t_{1}$ is the time the last person entered, and $t_{2}$ is the time the first person entered.

The times between the first and last entrance were $2 \sim 16$ seconds. The densities of people were $1.0 \sim 1.5$ people $/ \mathrm{m}^{2}$ for $\mathrm{P} 1$ and $\mathrm{P} 1$; $; 2.0 \sim 2.5$ people $/ \mathrm{m}^{2}$ for P2; $2.0 \sim 3.0$ people $/ \mathrm{m}^{2}$ for P3 as observed from a study of the video recordings.

The flow at the entrance of the staircase in each case is shown in Fig. 3. Figure 3 shows the flow of each P1 case is greater than each P2 case under the same conditions, except the case of merging from side "G" with the door closed. Also, the flow of each P2 case is bigger than that of each P3 for identical conditions. Therefore, it can be said the relationship between the ease of merging and the staircase population density is that merging is easier when the density is lower.
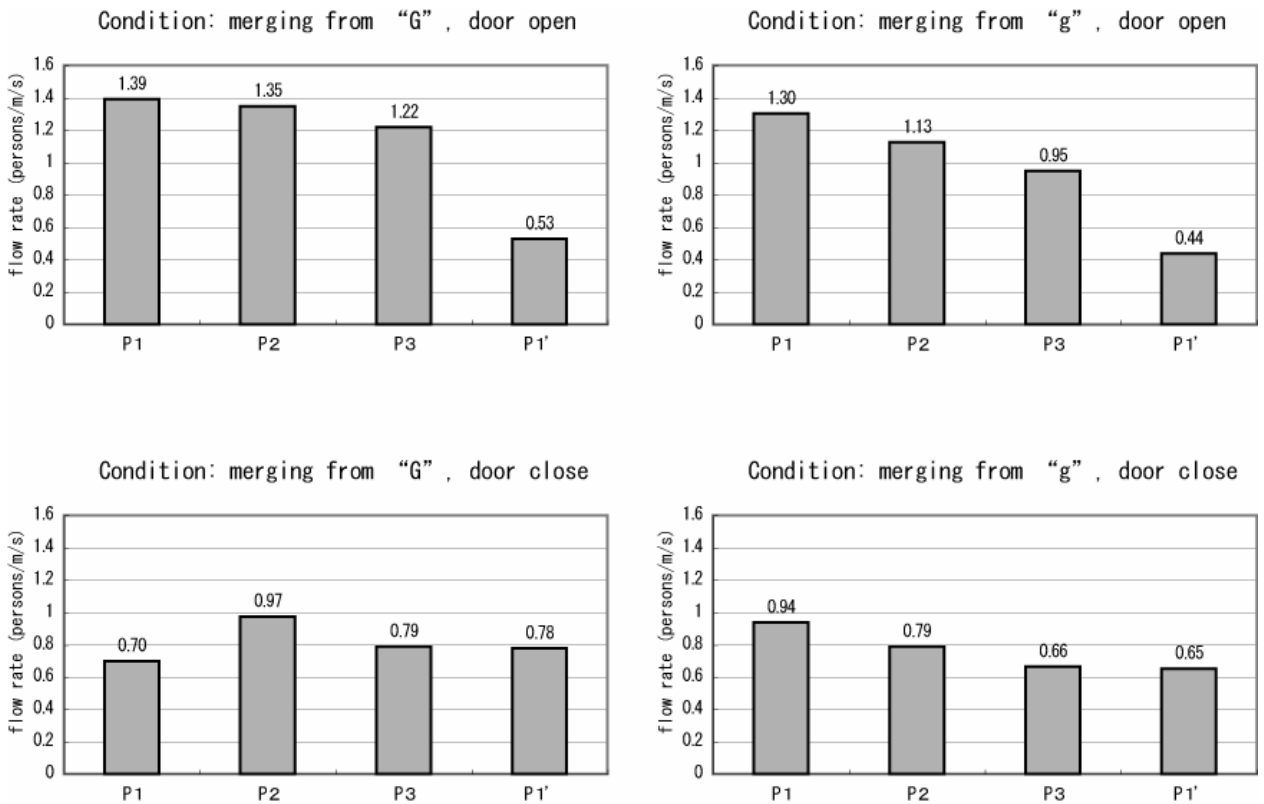

Fig. 3. Flows entering the staircase. 


\section{Relationship between Flow and Direction of Merging}

Figure 4 shows the relationship between flow and merge direction. The upper graph shows the case where the door was initially opened; the lower graph shows case where the door was initially closed. Comparison of densities shows that in every case except P1 merging from side " $G$ " with the door closed, the flow merging from the opposing side "g" is about 15-20\% lower than the flow merging from following side "G." Therefore, it can be said that the relationship between the ease of merging and the direction of merge is that merging is easier when the merging group moves in the same direction as the group already on the staircase.

Condition: Door Open

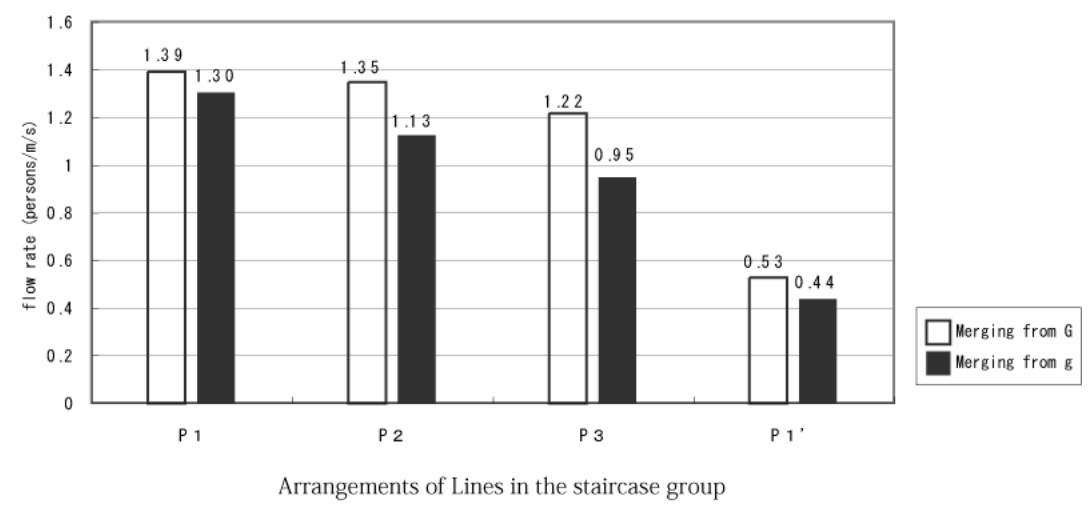

Condition: Door Close

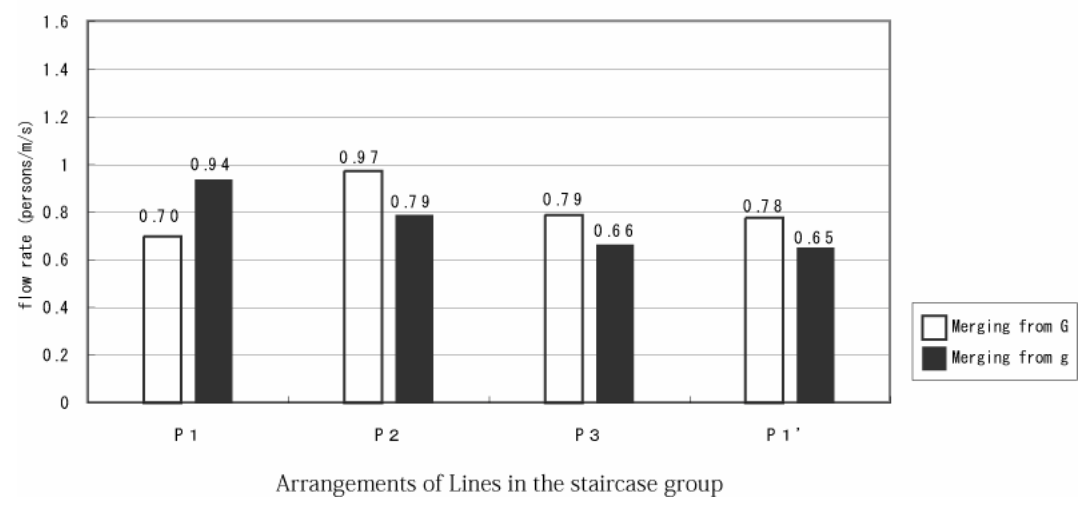

Fig. 4. Relationship between flow and merging direction.

\section{Relationship between Flow and Door State}

Figure 5 shows the relationship between flow and door state. The upper graph shows the result of merging from following side "G"; the lower graph shows the result of merging from opposing side "g". The flow when the door is closed is about $30 \%$ lower than the flow when the door is opened. As with the comparison of density and direction of merge, in any case except P1 merging from side "G" with door closed, flow from opposing "g" 
is lower than from following "G." Therefore, it can be said that the relationship between the ease of merging and the door state is that merging is easier when door is initially opened.

Condition: Merging from " $G$ "

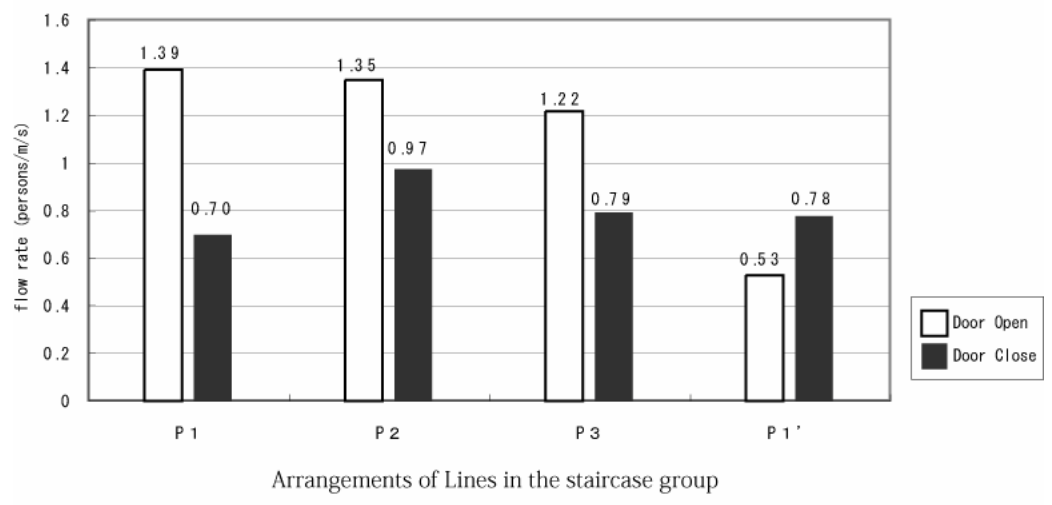

Condition: Merging from "g"

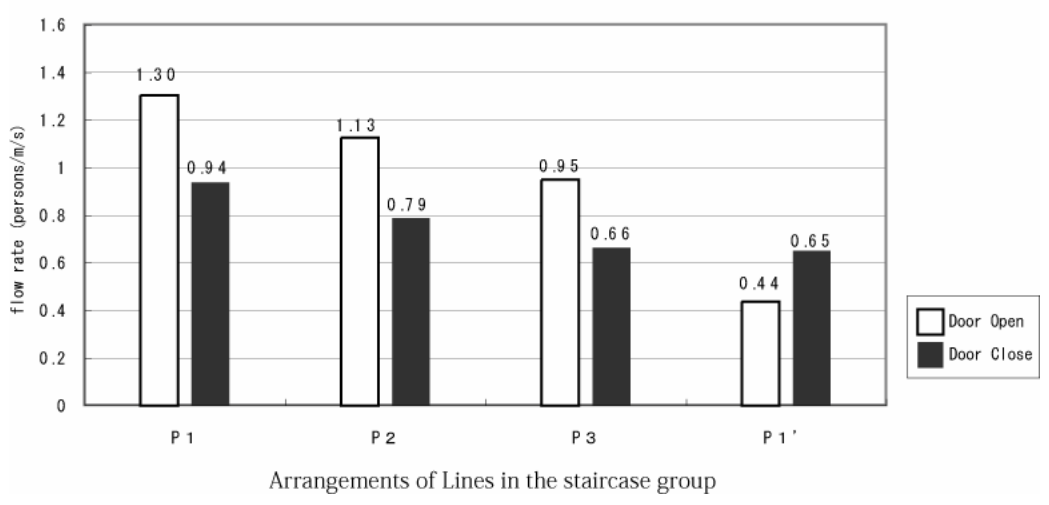

Fig. 5. Relationship between flow and door state.

\section{DISCUSSION}

Figure 3 reveals that in the case of merging from following side with the door closed, the flow of P1 remains lower than those of P2 and P3. Also, in Figs. 4 and 5, the flow of the P1 exhibits different characteristic behavior than the others. To analyze the reason behind this reversal of order, we focused on the position of the door.

Figure 6 shows the angle of door opening during the various tests with merging from the following side "G" when the door was initially closed. We can see that the door angle was smaller for case P1 than for P2 and P3. This caused the differences in the effective opening width for passing and therefore the reversed flow trend.

In other words, for cases P2 and P3, the door is fully opened. However, at a low staircase density such as that of P1 where the door is initially closed, merging occurs without the 
door being fully opened; thus, it may take more time to merge compared to cases in which the density of the staircase group is higher.

In the cases of merging from the opposite side with the door initially closed, the door never opened fully in any case as indicated in Fig. 7. Therefore, the flow was lower than the cases of merging from the following side.

In these experiments, subjects were all "healthy" students. In real society, there exists a variety of people, exhibiting variety in age, mobility, etc. To focus this analysis on the built environment, simple subjects were used. However, we think it is necessary to perform other experiments with varying human characteristics to generalize the results to real evacuation.

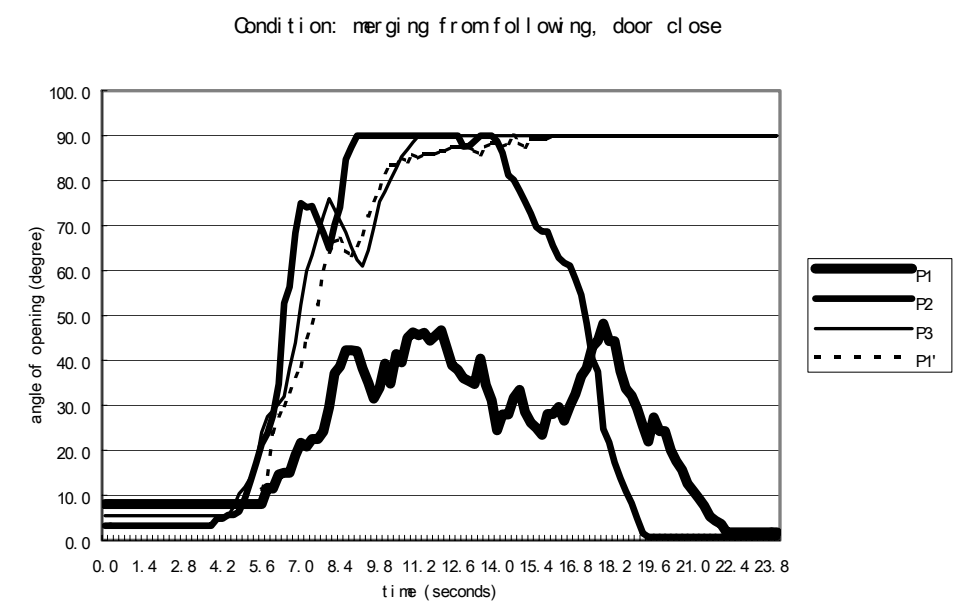

Fig. 6. Angle of door opening when merging occurs at the following side.

Conditi on: merging from opposi te si de, door cl ose

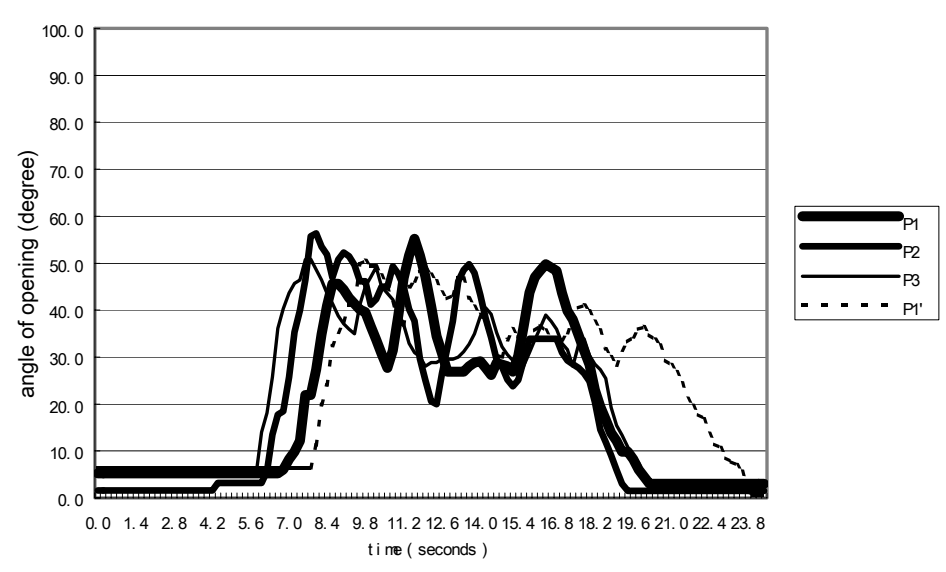

Fig. 7. Angle of door opening when merging occurs at the opposing side. 


\section{CONCLUSION}

Regarding the relationship between the ease of merging and the staircase population density, merging is easier when the density is lower.

The flow when merging occurs opposing stair traffic is about $15-20 \%$ lower than the flow when merging is oriented with stair traffic. Therefore, merging is easier when the merging group follows the staircase group.

The flow when the door leading to the staircase is initially closed is about $30 \%$ lower than the when the door is initially opened. Therefore, it is easier to merge when door is initially open.

\section{REFERENCES}

[1] Hokugo, A., Kubo, K., and Murozaki, Y, "An Experimental Study on Confluence of Two Foot Traffic Flows in Staircase," Journal of Architecture, Planning and Environmental Engineering, No.358, Architectural Institute of Japan, pp. 37-43, Dec., 1985.

[2] Hagiwara, I., "Evaluation Method of Egress Safety,” 15th US-Japan on Natural Resources Panel on Fire Research and Safety, Vol. 1, March, 2000, pp. 161165.

[3] Proulx, G., and Fahy, R.F., "Evacuation of the World Trade Center: What Went Right?," Proceedings of the CIB-CTBUH International Conference on Tall Buildings, CIB Publications No. 290, 2003, pp. 27-34.

[4] Kuligowski, E.D., and Milke, J.A., "A Performance-based Design of a Hotel Building Using Two Egress Models: A Comparison of the Results," Human Behaviour in Fire Symposium, 2004, pp. 399-410. 